\title{
Алгоритм моделирования двухкомпонентной динамики сорбции в случае смешанной дифффузионной кинетики
}

\author{
Прудковский А.Г. \\ Институт геохимии и аналитической химии им. В.И. Вернадского Российской Академии наук, \\ Москва
}

Поступила в редакцию 25.11.2017 г.

Описан алгоритм моделирования процесса двухкомпонентной динамики сорбции смешенной диффузионной кинетики, с граничным условием, удовлетворяющим соотношениям Никольского. Алгоритм может быть использован при создании программы для решения обратной задачи по определению коэффициентов внешней и внутренней диффузии, а также константы обмена.

Ключевые слова: Математическая модель, алгоритм, сорбция, диффузия, кинетика, обратная задача.

\section{Algorithm for modeling two-component sorption dynamics in the case of mixed diffusion kinetics}

\author{
Prudkovskii A.G. \\ Vernadsky Institute of Geochemistry and Analytical Chemistry of Russian Academy of Sciences (GEOKHI \\ RAS). Moscow
}

In 1981, Mark Moiseevich Senyavin tasked with determining the ion-exchange equilibrium and kinetic coefficients of strontium and ammonium ions from low-mineralized waters on clinoptilolite-containing tuffs of various deposits in Russia. As a result, a group of employees of the laboratory of sorption methods of GEOKHI created an experimental stand to calculate the output curves of sorption, as well as software for determining the coefficients of internal and external diffusion and the constants of constant exchange of sorbents.

The disadvantage of the work carried out is that the calculations were carried out using simplified formulas, either for external or internal diffusion, while should be considered the possibility of mixed diffusion should be taken into account. The time has passed for which the power of computing devices has multiplied. Now the problem of sorption can be solved directly, with the help of a numerical calculation. The advantage of this approach is that there is no need to impose any additional conditions on the task. For example, there is no need to consider a column completely washed before the start of the experiment, if desired, you can specify a column filling and, optionally, this filling should be uniform along the length of the column.

In this paper, we describe an algorithm for the two-component sorption dynamics in the case of mixed diffusion kinetics. Previously, we created the "CreateScheme" program, which is capable of calculating a multicomponent sorption process. However, the program does not allow us to investigate the subtleties of the sorption dynamics, since it uses rather large time steps to calculate the diffusion process according to equilibrium formulas. This article describes an algorithm that describes the process of mixed diffusion with smaller steps in time. The main difficulty was the docking of the first-order external diffusion equation with the second-order internal diffusion equation. This coupling was achieved with the help of the original method of replacing the second-order internal diffusion equation near the grain surface of the sorbent by an equation of the first order. The algorithm is implemented as a "DifFind" program, which is currently in the process of debugging.

Keywords: Mathematical model, algorithm, absorption, diffusion, kinetics, inverse problem. 


\section{Введение}

В 1981 году Марком Моисеевичем Сенявиным была поставлена задача по определению ионообменных равновесных и кинетических коэффициентов ионов стронция и аммония из маломинерализованных вод на клиноптилолитсодержащих туфах различных месторождений России: Шивыртуйское (Сибирь), Холинское, Чугуевское (Приморский край), Ягоднинское (Камчатка), Татарско-Шатрашанское (Татарстан) и Погребское (Брянская область). Для этой цели был создан сорбционный стенд с использованием автоматизированной системы АСНИ ГЕОХИ. Полученные на стенде выходные кривые сорбции стронция [1] и аммония [2] для всех изученных месторождений внесены в банк экспериментальных выходных кривых для данной системы. Методом решения обратных задач [3] для каждой выходной кривой были определены равновесный и кинетический коэффициенты. Решения обратных задач были рассчитаны на основании линейной или квадратичной изотерме с преобладанием либо внешне, либо внутри-диффузионной кинетики $[4,5]$, хотя сравнением критериев, полученных при решении обратных задач для разных математических моделей, было показано, что обмен иона аммония на клиноптилолитах в рассматриваемой системе отвечает динамике обмена при смешаннодиффузионной кинетике.

Прошло время, за которое мощь вычислительных устройств многократно возросла. Теперь задача сорбции вполне может решаться напрямую, с помощью численного счёта. Преимущество такого подхода в том, что не надо накладывать никаких дополнительных условий на задачу. Например, нет необходимости считать колонку полностью отмытой перед началом эксперимента, при желании можно задать то или иное заполнение колонки и необязательно это заполнение должно быть равномерным по длине колонки.

Так, нами была создана программа «CreateScheme» $[6,7]$ для конструирования, расчета и визуализации ионообменных технологических схем. Программа способна рассчитывать многокомпонентный процесс сорбции, а также регенерации и отмывки в нескольких колонках, связанных в технологическую схему. Однако, программа «CreateScheme» не позволяет исследовать тонкости динамики сорбции, так как при расчётах использует достаточно большие шаги по времени, которые рассчитываются по ориентировочным значениям коэффициентов внешней и внутренней диффузии.

В данной работе описан алгоритм моделирования процесса двухкомпонентной динамики сорбции при смешанной диффузии с граничным условием, удовлетворяющим соотношениям Никольского, который может быть применён для решения обратной задачи по определению коэффициентов внешней и внутренней диффузии.

\section{Теоретическая часть}

Предполагается, что жидкая фаза содержит ионы первого вещества с зарядом $z_{1}$ в концентрации $\left.z_{1} c_{1}\right|_{x=0}=C_{0}$, которое обменивается на сорбенте объёмом $\left.z_{2} c_{2}\right|_{t=0}=A_{0}$, полностью заполненным ионами второго вещества с зарядом $z_{2}$. Концентрации веществ достаточно велики, чтобы можно было пренебрегать концентрациями ионов $\mathrm{H}^{+}$и $\mathrm{OH}^{-}$. Движение вещества по колонке описывается уравнениями переноса:

$$
\left(\frac{\partial c_{k}}{\partial t}+\frac{v}{\varepsilon} \frac{\partial c_{k}}{\partial x}\right)+\varepsilon_{1} \frac{\partial a_{k}}{\partial t}=D_{l} \frac{\partial^{2} c_{k}}{\partial x^{2}} ; \quad c_{k}=c_{k}(x, t) ; a_{k}=a_{k}(x, t)
$$




$$
a_{k}=\frac{3}{R^{3}} \int_{0}^{R} u_{k} r^{2} d r ; u_{k}=u_{k}(x, t, r) ; \varepsilon_{1}=\frac{1-\varepsilon}{\varepsilon},
$$

где $v$ - линейная скорость жидкой фазы на входе в колонку, $\varepsilon$ - доля жидкой фазы в колонке, а $\varepsilon_{1}$ - отношение объёмов твёрдой и жидкой фаз в колонке, $c_{k}, a_{k}, k=1,2$ концентрации ионов в жидкой фазе и в сорбенте, $D_{l}$ - коэффициент продольной диффузии. Предполагается, что сорбент состоит из шаров, диаметром $R$, где $u_{k}=u_{k}(x, t, r)$ - концентрация ионов внутри шара. Обмен ионов на поверхности зёрен сорбента удовлетворяет уравнениям Никольского:

$$
\left(\frac{u_{1}(x, t, R)}{c_{1 R}(x, t)}\right)^{1 / z_{1}}=K\left(\frac{u_{2}(x, t, R)}{c_{2 R}(x, t)}\right)^{1 / z_{2}}
$$

где $K$ - константа обмена. Предполагая, что ёмкость сорбента постоянна $A_{0}$, получим, что и общая концентрация ионов в жидкой фазе тоже постоянна $C_{0}$. Уравнения электронейтральности:

$$
z_{1} c_{1}+z_{2} c_{2}=C_{0} ; z_{1} a_{1}+z_{2} a_{2}=A_{0} .
$$

Обмен ионов между жидкой фазой и сорбентом описывается уравнениями внешней и внутренней диффузии:

$$
\begin{gathered}
\frac{\partial c_{1}}{\partial t}=\beta\left(c_{1 R}-c_{1}\right), \\
\frac{\partial u_{1}}{\partial t}=D \frac{1}{r^{2}} \frac{\partial}{\partial r}\left[r^{2} \frac{\partial u_{1}}{\partial r}\right] ; u_{1_{r=R}}=u_{1 R} ;{\frac{\partial u_{1}}{\partial r}}_{r=0}=0 .
\end{gathered}
$$

где коэффициент $\beta$ управляет внешней диффузией, а коэффициент $D$ - внутренней диффузией. Все коэффициенты $D_{l}, K, \beta, D$ предполагаются постоянными.

Особенности процесса сорбции тесно связаны с тремя характерными временными параметрами: $\Delta t_{\beta}=\beta^{-1}$ - характерное время внешней диффузии, $\Delta t_{D}=\frac{R^{2}}{D}$ характерное время внутренней диффузии и $\Delta t_{l}=\varepsilon^{2} \frac{D_{l}}{v^{2}}$ - характерное время продольной диффузии. В зависимости от соотношения между этими параметрами кинетику сорбции относят: либо к внешнедиффузионной $\Delta t_{\beta}>\Delta t_{D}$, либо к внутридиффузионной $\Delta t_{\beta}<<t_{D}$, либо кинетика процесса считается смешанной.

Для послойного счёта необходимо выбрать шаг по времени $\Delta t$, определяющий величину порции, и шаг по длине колонки $\Delta x$, определяющий величину слоя. Если для алгоритма «CreateScheme» [7] эти параметры выбирались достаточно большими, чтобы превысить характерные времена диффузии, то в данной программе они выбираются в соответствии с необходимой точностью решения задачи, хотя есть одно «техническое» условие - длина порции $\Delta L$ в жидкой фазе кратна ширине слоя сорбента $\Delta x$ :

$$
\Delta L=\Delta t \frac{v}{\varepsilon}=N_{t x} \Delta x \quad \text { или } \frac{\Delta L}{\Delta x}=N_{t x}
$$

где $N_{t x}>0$ - некоторое целое число, при $N_{t x}=1$ получаем привычную послойную модель расчёта. Итак, разностная схема оперирует сорбентом в колонке, разделённом на слои длиной $\Delta x: x_{i}, i=1, \ldots N_{x}, x_{1}=0, x_{N_{x}}=L$, где $L$ - длина колонки; а также жидкой фазой, разделённой на слои, длиной $\Delta L=\Delta t \frac{v}{\varepsilon}=N_{t x} \Delta x$, которые можно за- 
нумеровать индексом $j=1, . ., N_{t}$. В результате, в каждом слое мы имеем концентрации ионов $a_{k i}(t)$, а в жидкой фазе имеем концентрации ионов $c_{k j}(x, t)$. фазе:

Первый шаг разностной схемы реализует продольную диффузию в жидкой

$$
c_{k j}(x, t+\widetilde{\Delta} t)=c_{k j}(x, t)+\widetilde{\Delta} t \frac{D_{l}}{\Delta L^{2}}\left(c_{k j-1}(x, t)-2 c_{k j}(x, t)+c_{k j+1}(x, t)\right)
$$

Если $\Delta t \frac{D_{l}}{\Delta L^{2}} \leq \frac{1}{3}$, то в качестве $\widetilde{\Delta} t$ используется основной шаг схемы $\Delta t$, иначе продольная диффузия проводится в несколько шагов $\widetilde{\Delta} t=\frac{\Delta t}{m}$, целое $\mathrm{m}$ выбирается так, чтобы выполнялось неравенство $\widetilde{\Delta} t \frac{D_{l}}{\Delta L^{2}} \leq \frac{1}{3}$. Для завершения шага по времени осталось реализовать уравнение переноса (1) с $D_{l}=0$ и провести взаимодействие с сорбентом по уравнениям $(5,6)$. Делаем замену переменных: $\tau=t-\frac{x}{v} \varepsilon$, подставляем в (1) при $D_{l}=0$ и получаем уравнение:

$$
\frac{v}{\varepsilon} \frac{\Delta c_{k}}{\Delta x}+\varepsilon_{1} \frac{\Delta a_{k}}{\Delta \tau}=0
$$

При каждом фиксированном $x=x_{i}$, происходит обмен между жидкой фазой и сорбентом, затем порция жидкой фазы передвигается на расстояние $\Delta x$, чтобы взаимодействовать со следующим слоем. Из нашей замены $\tau=t-\frac{x}{v} \varepsilon$ следует, что при фиксированной координате $x$ приращения $\tau$ и $t$ равны $\Delta \tau=\Delta t$, так что при описании взаимодействий жидкой порции со слоем будем использовать вместо $\Delta \tau$ величину $\Delta t$. Таким образом, уравнение (9) можно переписать в виде:

$$
V_{m}\left(c_{k j+1}-c_{k j}\right)=-\left(a_{k i+1}-a_{k i}\right) ; V_{m}=\frac{v \Delta t}{\varepsilon \varepsilon_{1} \Delta x}=\frac{\Delta L}{\Delta x \varepsilon_{1}}=\frac{N_{t x}}{\varepsilon_{1}} .
$$

\section{Описание смешанной диффрузии}

Каждая ј-ая порция при первом касании взаимодействует с і-ым слоем в течении шага времени $\Delta t$. При дальнейшем описании этого взаимодействия индексы $\mathrm{i}$, j опускаем, также и зависимость от пространственной переменной $x$. Итак, уравнение (10) можно переписать в более удобной для расчётов форме:

$$
V_{m} c_{k}+a_{k}=Q_{k}=\text { const }
$$

Если шаг по времени $\Delta t$ меньше характерного времени диффузии $\Delta t \leq \Delta t_{D}=\frac{R^{2}}{D}$, то делим зерно сорбента на целое число слоёв толщиной $\Delta r \approx \sqrt{\Delta t D}$. Тогда за время $\Delta t$ жидкая фаза успеет взаимодействовать только с верхней частью зерна, объёмом $\frac{4}{3} \pi\left[R^{3}-(R-\Delta r)^{3}\right]=\frac{4}{3} \pi R^{3}\left[1-\left(1-\frac{\Delta r}{R}\right)^{3}\right]$, что составляет $\chi$ - тую долю общего объёма сорбента:

$$
\chi=\left[1-\left(1-\frac{\Delta r}{R}\right)^{3}\right]
$$

При $\Delta r=R$ получаем $\chi=1$, а при $\Delta r \rightarrow 0 \quad \chi \rightarrow 3 \frac{\Delta r}{R}$. 
Уравнение (11) в этом случае перепишется в виде:

$$
V_{m \chi} c_{k}+u_{k \chi R}=Q_{k \chi}=\text { const, }
$$

где через $u_{k \chi R}$ обозначена средняя концентрация k-ого компонента в слое, толщиной $\Delta r$ у поверхности зерна сорбента (при $r \in[R-\Delta r, R]), V_{m \chi}=\frac{V_{m}}{\chi}$.

Теперь, считая, что наш шаг по времени приблизительно равен характерному времени внутренней диффузии $\Delta t \approx \Delta t_{D \chi}=\frac{\Delta r^{2}}{D}$, уравнение внутренней диффузии (6) на поверхности сорбента можно упростить (проведена численная проверка):

$$
\frac{\partial u_{1 \chi R}}{\partial t}=\gamma\left(u_{1 R}-u_{1 \chi R}\right) ; \gamma=\frac{D}{R^{2}} \frac{3}{\chi\left[1-\sqrt[3]{1-\frac{\chi}{2}}\right]},
$$

где $u_{k R}=u_{k}\left(x_{i}, t, R\right)$. При $\Delta r=R$ получаем $\gamma=\frac{D}{R^{2}} \frac{3 \sqrt[3]{2}}{\sqrt[3]{2}-1} \approx 14,54 \frac{D}{R^{2}}$, а при $\Delta r \rightarrow 0$ величина $\gamma \rightarrow 2 \frac{D}{\Delta r^{2}}$. Перепишем теперь соотношение (3), используя (4), в виде:

$$
\left(\frac{u_{1 R}}{c_{1 R}}\right)^{1 / z_{1}}=K\left(\frac{u_{2 R}}{c_{2 R}}\right)^{1 / z_{2}} \Rightarrow \Gamma^{\frac{z_{2}}{z_{1}}}\left[C_{0}-z_{1} c_{1 R}\right]=K^{z_{2}}\left[A_{0}-z_{1} \Gamma c_{1 R}\right]
$$

где $\Gamma=\frac{u_{1 R}}{c_{1 R}}=\frac{u_{1}\left(x_{i}, t, R\right)}{c_{1 R}\left(x_{i}, t\right)}$. Соотношение (15) устанавливает связь между коэффициентом распределения $\Gamma$ сорбируемого компонета $c_{1}$ и концентрацией этого компонента $c_{1 R}$ на поверхности зерна сорбента. Предположим, что в течение времени взаимодействия $\Delta t$ величина $\Gamma$ меняется достаточно мало, чтобы её можно было считать константой. Если это не так, то придётся данный шаг $\Delta t$ делить на более мелкие шаги.

Учитывая закон сохранения вещества (13) при его сорбции в любом і-ом слое, имеем:

$$
\frac{\partial}{\partial t}\left(V_{m \chi} c_{1}+u_{1 \chi R}\right)=0
$$

Из уравнений (16), (5), (14) имеем:

$$
V_{m \chi} \beta\left(c_{1 R}-c_{1}\right)+\gamma\left(u_{1 R}-u_{1 \chi R}\right)=0 \Rightarrow V_{m \chi} \beta\left(c_{1 R}-c_{1}\right)+\gamma\left(\Gamma c_{1 R}-u_{1 \chi R}\right)=0
$$

Из (17) находим величину $c_{1 R}$

$$
c_{1 R}=\frac{V_{m \chi} \beta c_{1}+\gamma u_{1 \chi R}}{V_{m \chi} \beta+\gamma \Gamma}
$$

и подставляем в соотношение (15):

$$
\Gamma^{\frac{z_{2}}{z_{1}}}\left[C_{0}-z_{1} \frac{V_{m \chi} \beta c_{1}+\gamma u_{1 \chi R}}{V_{m \chi} \beta+\gamma \Gamma}\right]=K^{z_{2}}\left[A_{0}-z_{1} \Gamma \frac{V_{m \chi} \beta c_{1}+\mu_{1 \chi R}}{V_{m \chi} \beta+\gamma \Gamma}\right],
$$

откуда можно найти зависимость константы обмена от концентраций вещества в жидкой фазе и в верхнем слое зерна сорбента $\Gamma\left(c_{1}, u_{1 \chi R}\right)$. Учитывая, что в силу малости шага по времени $\Delta t$ концентрации в жидкой фазе и в сорбенте меняются мало, можно считать, что величина $\Gamma\left(c_{1}, u_{1 \chi R}\right)$ приблизительно равна константе и её можно вычислять по значениям $c_{1}, u_{1 \chi R}$ в начальный момент времени, а потом уточнять по результирующим значениям, которые ещё предстоит найти, в конце сорбции. В слу- 
чае, если на каком-то отрезке счёта величина $Г\left(c_{1}, u_{1 \chi R}\right)$ быстро меняется, то на этом отрезке можно производить расчёт смешенной диффузии с более мелким шагом.

Выведем теперь уравнение смешанной диффузии из уравнений (5), (18) (13):

$$
\begin{aligned}
\frac{\partial c_{1}}{\partial t} & =\beta\left(c_{1 R}-c_{1}\right) \Rightarrow \frac{\partial c_{1}}{\partial t}=\beta\left(\frac{V_{m \chi} \beta c_{1}+\mu_{1 \chi R}}{V_{m \chi} \beta+\gamma \Gamma}-c_{1}\right) \Rightarrow \frac{\partial c_{1}}{\partial t}=\frac{\beta \gamma}{V_{m \chi} \beta+\gamma \Gamma}\left(u_{1 \chi R}-\Gamma c_{1}\right) \\
& \Rightarrow \frac{\partial c_{1}}{\partial t}=\frac{\beta \gamma}{V_{m \chi} \beta+\gamma \Gamma}\left[Q_{1 \chi}-c_{1}\left(V_{m \chi}+\Gamma\right)\right] \Rightarrow \frac{\partial c_{1}}{\partial t}=\frac{\beta \gamma\left(V_{m \chi}+\Gamma\right)}{V_{m \chi} \beta+\gamma \Gamma}\left[\frac{Q_{1 \chi}}{V_{m \chi}+\Gamma}-c_{1}\right]
\end{aligned}
$$

Уравнение (20) позволяет совершить шаг по времени:

$$
c_{i}(t+\Delta t)=c_{i}(t) e^{-\widetilde{\beta} \Delta t}+\frac{Q_{1 \chi}}{V_{m \chi}+\Gamma}\left(1-e^{-\widetilde{\beta} \Delta t}\right)
$$

где $\widetilde{\beta}=\frac{\beta \gamma\left(V_{m \chi}+\Gamma\right)}{V_{m \chi} \beta+\gamma \Gamma}$. Из уравнения (13) находим среднюю концентрацию первого компонента в верхнем слое зерна:

$$
u_{1 \chi R}(t+\Delta t)=Q_{1 \chi}-V_{m \chi} c_{1}(t+\Delta t)
$$

Если $\Delta t \geq \Delta t_{D}=\frac{R^{2}}{D}$, то нет необходимости делить зерно на слои и верхний слой совпадает со всем зерном, так что $\chi=1$ и $a_{1}(t+\Delta t)=u_{1 \chi R}(t+\Delta t)$, иначе придётся проводить расчёт внутренней диффузии (6) с помощью разностной схемы. Заменой переменных $\tau_{R}=\left(t-t_{i}\right) \frac{D}{R^{2}}, z=\frac{r}{R}$ преобразуем уравнение (6) к виду:

$$
\frac{\partial u_{1}}{\partial \tau_{R}}=\frac{1}{z^{2}} \frac{\partial}{\partial x}\left[z^{2} \frac{\partial u_{1}}{\partial x}\right] ; u_{1}(z, 0)=u_{10}(z) ;\left.\frac{\partial u_{1}\left(z, \tau_{R}\right)}{\partial z}\right|_{z=0}=0 ; z \in[0,1]
$$

В разностной схеме учитываем, что начальное значение концентрации в верхнем слое зерна у нас уже найдено в (21), хотя оно ещё изменится в процессе внутренней диффузии. Произведём замену:

$$
w\left(z, \tau_{R}\right)=z\left[u_{R}-u_{1}\left(R z, \tau_{R} \frac{R^{2}}{D}\right)\right],
$$

где $u_{R}=u_{1 \chi R}(t+\Delta t)$. Таким образом, мы приводим уравнение к стандартному виду:

$$
\frac{\partial w}{\partial \tau_{R}}=\frac{\partial^{2} w}{\partial z^{2}} ; w(x, 0)=z u_{R}(t+\Delta t)-z u_{0}(R z) ; w\left(1, \tau_{R}\right) \equiv 0 ;\left.\frac{\partial}{\partial z}\left(\frac{w\left(z, \tau_{R}\right)}{z}\right)\right|_{z=0}=0
$$

и решаем с помощью стандартной разностной схемы.

$$
\begin{array}{r}
w(t+\widetilde{\Delta} t)=w(t)+\frac{\Delta \tau}{\Delta z^{2}}(w(z-\Delta z)-2 w(z)+w(z+\Delta z)) ; \\
\frac{\Delta \tau}{\Delta z^{2}} \leq \frac{1}{3} ; \Delta \tau \leq \frac{\Delta z^{2}}{3} ; \tilde{\Delta} t \frac{D}{R^{2}} \leq \frac{\Delta r^{2}}{3 R^{2}} ; \widetilde{\Delta} t \leq \frac{\Delta r^{2}}{3 D}
\end{array}
$$

Учитывая, что шаг по зерну $\Delta r \approx \sqrt{\Delta t D}$, получаем:

$$
\Delta t \approx \frac{\Delta r^{2}}{D} \Rightarrow \Delta t \approx 3 \widetilde{\Delta} t
$$

То есть, необходимо три шага по схеме (25), чтобы обеспечить шаг $\Delta t$. Получив распределение концентраций $u_{1}(t+\Delta t, r)$, вычисляем среднюю концентрацию $a_{1}(t+\Delta t)$ в зерне сорбента по формуле (2), а затем корректируем значение концентрации того же компонента в жидкой фазе по формуле (11). Концентрации второго 
компонента находятся как дополнительные из соотношений электронейтральности (4). Произведя подобные действия со всеми порциями, находящимися в колонке, можно все их передвинуть на шаг $\Delta x$ и повторить те же расчёты взаимодействия со следующими слоями сорбента.

\section{Эксперимент}

Алгоритм, описанный в данной работе был реализован в виде компьютерной программы DifFind [8]. Главное окно программы представлено на рис.1. Нижняя кривая представляет собой экспериментальную кривую, а верхняя - теоретическая кривая, найденная автоматическим поиском значений коэффициентов диффузии и константы обмена. На рис.1 программа DifFind определила два коэффициента: коэффициент внутренней диффузии $2.110^{-7}$ сек $^{-1}$ и константу обмена Никольского 1.2. Пока точность работы программы $10 \%$.

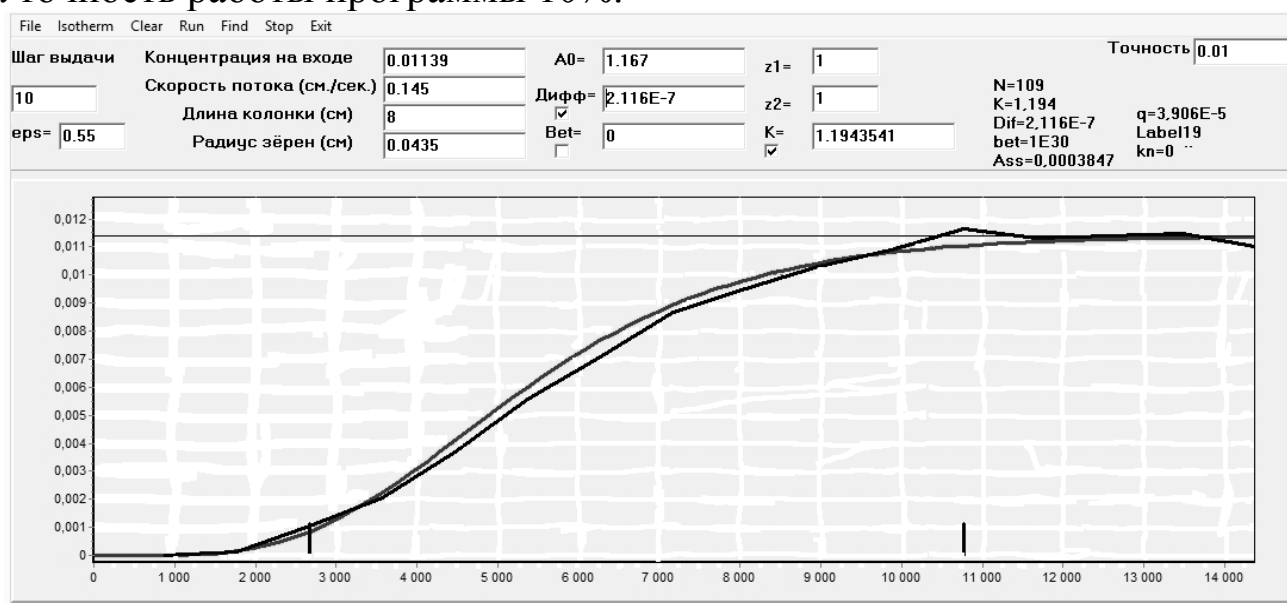

Рис. 1 Главное окно программы DifFind. Колонка в натриевой форме, длиной $8 \mathrm{~cm}$., сечение $1.56 \mathrm{~cm}^{2}$, диаметр зёрен сорбента $0.087 \mathrm{~cm}$., порозность 0.55 , тип сорбента «jgodnin», жидкая фаза - хлорид аммония, скорость потока $0.1451 \mathrm{~cm} /$ сек, продолжительность стадии 13500 сек.

\section{Заключение}

Программа DifFind, реализующая описанный здесь алгоритм в настоящее время находится в процессе отладки. В основном, стоят проблемы оптимизации алгоритма по точности и быстродействию. Возможны также ошибки работы программы в случае больших градиентов коэффициента распределения при обмене разнозарядных ионов. Всё это ещё предстоит исследовать в будущем.

Автор выражает благодарность Комаровой И.В. за полезные обсуждения.

\section{Список литературы}

1. Галкина Н.К.,. Комарова И.В., Никашина В.А. Анфилов Б.Г. // Сорбиионные и хроматографические прочессы. 2001. Т. 1. № 3.C. 483-487.

2. Комарова И.В., Галкина Н.К., Никашина В.А., Анфилов Б.Г. и др. // Сорбиионные и хроматографические прочессы. 2001. Т. 1. № 3. C. 490-494.

3. Комарова И.В., Галкина Н.К.,. Никашина В.А. Анфилов Б.Г. // Сорбиионные и хроматографические проиессы. 2001. Т. 1. № 4. C. 606-612. 
4. Жуховицкий А.А., Забежинский Я.Л., Тихонов А.Н. // Журнал Физ. Химии. 1945. Т. 19. № 6. C. 253-261.

5. Веницианов Е.В., Рубинштейн Р.Н. Динамика сорбции из жидких сред. М. Наука. 1983. $237 \mathrm{c}$.

6. Прудковский А.Г., Комарова И.В., Галкина Н.К. // Сорбиионные и хроматографические прочессы. 2014. Т. 14. № 5. С. 824831.

\section{References}

1. Galkina N.K.,. Komarova I.V., Nikashina V.A. Anfilov B.G., Sorbtsionnye $i$ khromatograficheskie protsessy, 2001, Vol. 1. No 3. pp. 483-487.(Rus)

2. Komarova I.V., Galkina N.K., Nikashina V.A., Anfilov B.G. et al., Sorbtsionnye i khromatograficheskie protsessy, 2001, T.1. № 3, S. 490-494. (Rus)

3. Komarova I.V., Galkina N.K.,. Nikashina V.A. Anfilov B.G., Sorbtsionnye i khromatograficheskie protsessy, 2001, Vol. 1, No 4, pp. 606-612. (Rus)

4. Zhuhovickij A.A., Zabezhinskij Ja.L., Tihonov A.N., Zhurnal Fiz. Himii, 1945, Vol. 19, No 6, pp. 253-261. (Rus)

5. Venicianov E.V., Rubinshtejn R.N., Dinamika sorbcii iz zhidkih sred, M., Nauka, 1983, 237 p. (Rus)

Прудковский Андрей Гаральдович - д.фм.н., старший научный сотрудник, лаборатория сорбционных методов, ГЕОХИ РАН,
7. Комарова И.В., Галкина Н.К., Прудковский А.Г., Хамизов Р.Х. // Сорбиионные и хроматографические прочессы. 2017. Т. 17. № 1. C.10-19.

8. Прудковский А.Г.. Материалы XV Международной научно-практической конференции, посвященной 115-летию открытия хроматографии и 100-летию Воронежского государственного университета Иониты. 2017. Воронеж. 13-17 сентября. С. 165-167.

6. Prudkovskij A.G., Komarova I.V., Galkina N.K., Sorbtsionnye $i$ khromatograficheskie protsessy, 2014, Vol. 14, No 5, pp. 824-831. (Rus)

7. Komarova I.V., Galkina N.K., Prudkovskij A.G., Hamizov R.H., Sorbtsionnye i khromatograficheskie protsessy, 2017, Vol. 17, No 1, pp. 10-19. (Rus)

8. Prudkovskij A.G.. Materialy XV Mezhdunarodnoj nauchno-prakticheskoj konferencii, posvjashhennoj 115-letiju otkrytija hromatografii i 100-letiju Voronezhskogo gosudarstvennogo universiteta , 2017, Voronezh, 13-17 .09, pp. 165-167(Rus)

Prudkovskii Andrei G. - Dr.Sci. (Phys/Math.), Leading researcher, Lab of Sorption Methods, GEOKHI RAS 